\title{
Rhizoma Dioscoreae extract protects against alveolar bone loss by regulating the cell cycle: A predictive study based on the protein-protein interaction network
}

\author{
ZHI-GUO ZHANG ${ }^{1}$, CHANG-HENG SONG ${ }^{1}$, FANG-ZHEN ZHANG $^{1}$, YAN-JING CHEN ${ }^{1}$, \\ LI-HUA XIANG ${ }^{1}$, GARY GUISHAN XIAO ${ }^{2,3}$ and DA-HONG JU ${ }^{1}$ \\ ${ }^{1}$ Institute of Basic Theory, China Academy of Chinese Medical Sciences, Beijing 100700; ${ }^{2}$ School of Pharmaceutical Science, \\ Dalian University of Technology, Dalian, Liaoning 116024, P.R. China; ${ }^{3}$ Functional Genomics and Proteomics Laboratory, \\ Osteoporosis Research Center, Creighton University Medical Center, Omaha 68131, NE, USA
}

Received November 5, 2015; Accepted February 2, 2016

DOI: $10.3892 / \mathrm{mmr} .2016 .5188$

\begin{abstract}
Rhizoma Dioscoreae extract (RDE) exhibits a protective effect on alveolar bone loss in ovariectomized (OVX) rats. The aim of this study was to predict the pathways or targets that are regulated by $\mathrm{RDE}$, by re-assessing our previously reported data and conducting a protein-protein interaction (PPI) network analysis. In total, 383 differentially expressed genes ( $\geq 3$-fold) between alveolar bone samples from the RDE and OVX group rats were identified, and a PPI network was constructed based on these genes. Furthermore, four molecular clusters (A-D) in the PPI network with the smallest P-values were detected by molecular complex detection (MCODE) algorithm. Using Database for Annotation, Visualization and Integrated Discovery (DAVID) and Ingenuity Pathway Analysis (IPA) tools, two molecular clusters (A and B) were enriched for biological process in Gene Ontology (GO). Only cluster A was associated with biological pathways in the IPA database. GO and pathway analysis results showed that cluster A, associated with cell cycle regulation, was the most important molecular cluster in the PPI network. In addition, cyclin-dependent kinase 1 (CDK1) may be a key molecule achieving the cell-cycle-regulatory function of cluster A. From the PPI network analysis, it was predicted that delayed cell cycle progression in excessive alveolar bone remodeling
\end{abstract}

Correspondence to: Professor Da-Hong Ju, Institute of Basic Theory, China Academy of Chinese Medical Sciences, 16 Nanxiaojie, Dongzhimennei, Beijing 100700, P.R. China

E-mail: judahong@sohu.com

Professor Gary Guishan Xiao, School of Pharmaceutical Science, Dalian University of Technology, 2 Linggong Road, Ganjingzi, Dalian, Liaoning 116024, P.R. China

E-mail: gxiao@dlut.edu.cn

Key words: cell cycle, herbal medicine, alveolar bone loss, ovariectomized rats, Rhizoma Dioscoreae, protein-protein interaction network, cyclin-dependent kinase 1 via downregulation of CDK1 may be another mechanism underling the anti-osteopenic effect of RDE on alveolar bone.

\section{Introduction}

Alveolar bone loss is associated with osteoporosis in postmenopausal women (1). It takes place rapidly during the early postmenopausal years prior to stabilization approximately six years after menopause, owing to a decline in estrogen levels (2). Estrogen (3), bisphosphonates (4) and parathyroid hormone (PTH) (5) have been employed to inhibit alveolar bone loss in postmenopausal women; however, overt adverse reactions can be caused by long-term treatment with these drugs (6-9). Thus, it is necessary to develop alternative therapeutic agents for preventing and treating alveolar bone loss.

In a previous study, it was demonstrated that Rhizoma Dioscoreae extract (RDE) has a protective effect on alveolar bone loss in ovariectomized (OVX) rats via regulation of Wnt and p38 mitogen-activated protein kinase (MAPK) signaling, using gene microarray and bioinformatics (10). Although the protective effect of RDE was partially explained, it was hypothesized that RDE could achieve its effect by regulating other signaling pathways or targets. Therefore, considering the important biological significance of protein-protein interaction (PPI) networks in the majority of molecular processes, our previous data (10) was analyzed with the predictive analysis method based on the PPI network to explore the potential pathways or targets regulated by RDE (11).

\section{Materials and methods}

Differentially expressed genes. As previously reported (10), our group demonstrated that RDE exhibits a protective effect on alveolar bone loss in OVX rats via regulation of Wnt and $\mathrm{p} 38$ MAPK signaling. A microarray analysis for gene expression profiling in alveolar bone samples from the two group rats was performed, and significant differentially expressed genes between two groups (cutoff of 3) were recorded. Bone 
tissue samples were obtained between the three molars and incisor of left mandibles. In the present study, the significant differentially expressed genes that were determined in our previous study were used. Methods of animal treatment, specimen preparation and microarray protocols are described in detail in our previous study (10). The present study was approved by the ethics committee of Institute of Basic Theory, China Academy of Chinese Medical Sciences (Beijing, China).

Construction of the PPI network. PPI represents an essential outline for the analysis of homeostasis and self-organization in living organisms. In cells or organisms, PPIs are important cellular events that form the basis for multiple signal transduction pathways or various transcriptional regulatory networks, further affecting the majority of molecular processes (12). Considering the importance of PPIs, a PPI network was established to explore the mechanisms underlying the effect of RDE on the prevention of bone loss.

In this study, BisoGenet (13), a Cytoscape (14) plugin (version 2.8.2), was used for building the PPI network. BisoGenet uses four databases to collect the information on PPI networks involving relevant genes. The four databases were BIND (Biomolecular Interaction Network Database; www.bind.ca), BioGRID (General Repository for Interaction Datasets; thebiogrid.org), DIP (Database of Interacting Proteins; dip.doe-mbi.ucla.edu/dip/), IntAct (Database system and analysis tools for protein interaction data; ebi.ac.uk/intact/), and MINT (Molecular Interactions Database; http://www.ebi. ac.uk/intact/). A PPI network was established based on the differentially expressed genes from microarray data analysis.

Detection of molecular clusters in the PPI network. Detecting molecular clusters in a PPI network is a common method to identify key biological functions in complex signaling pathways.

The database was integrated and the PPI network was generated an analyzed using the MCODE plugin in Cytoscape to analyze the PPI network features. MCODE can execute molecular complex detection (MCODE) algorithm to detect molecule clusters in the interactome network (15). Molecule clusters with $\mathrm{P}<0.05$, with at least four nodes and one seed node were considered significant.

Functional annotation of molecular clusters in the PPI network. Database for Annotation, Visualization, and Integrated Discovery (DAVID) Bioinformatics Resources 6.7 (16), a versatile functional annotation tool for discovering the biological significance of genes, was used to obtain biological process information of gene ontology $(\mathrm{GO})$ for genes in the PPI Network's molecular clusters, with a threshold EASE score set at 0.1 . In the analysis, enriched GO terms were considered to be statistically significant with $\mathrm{P}<0.05$ and false discovery rate $($ FDR $)<0.1$.

Pathway analysis of molecular clusters in the PPI network. To perform a pathway analysis, the names of proteins in clusters were uploaded into IPA (Ingenuity Systems, Redwood City, CA, USA). Based on two parameters, the most significant canonical pathways associated with the dataset were identified by IPA: i) Ratio of the number of proteins mapped to a pathway divided by the total number of proteins in the given canonical pathway and (ii) a P-value showing a degree of association between the canonical pathway and the dataset proteins.

Western blotting analysis. Western blotting was performed to validate protein expression of key genes in clusters. The rat alveolar bone, extracted as previously reported (10), of three groups (SHAM group, $n=6$; OVX group, $n=6$; RDE group, $\mathrm{n}=6$ ) were used in western blotting. After alveolar bone was homogenized in liquid nitrogen, proteins were extracted and dissolved in radioimmunoprecipitation assay buffer (Thermo Fisher Scientific, Inc., Waltham, MA, USA) containing phosphatase and protease inhibitors. The insoluble constituents were removed by centrifugation at $5,000 \mathrm{x}$ g for $5 \mathrm{~min}$ at $4^{\circ} \mathrm{C}$. Protein concentrations were determined with the bicinchoninic acid assay reagent (Pierce, Rockford, IL, USA). Total protein $(80 \mu \mathrm{g})$ were resolved by electrophoresis on $12 \%$ sodium dodecyl sulfate-polyacrylamide gels (Bio-Rad Laboratories, Inc., Hercules, CA, USA) and transferred onto nitrocellulose membranes (Hybond-ECL; GE Healthcare, Piscataway, NJ, USA). Membranes were blocked with $5 \%$ non-fat dry milk for $1 \mathrm{~h}$, incubated overnight at $4^{\circ} \mathrm{C}$ with antibodies against the following: Rabbit anti-rat polyclonal antibody against cyclin-dependent kinase 1 (CDK1; cat no. ab47594; 1:1,000 dilution) (Abcam, Cambridge, MA, USA) and rabbit anti-rat polyclonal antibody against $\beta$-actin (cat no. A2066; 1:25,000 dilution; Sigma-Aldrich, St. Louis, MO, USA). Subsequently, membranes were washed and incubated for $1 \mathrm{~h}$ with horseradish peroxidase-linked antibody (1:1,000 dilution, Cell Signaling Technology, Inc., Beverly, MA, USA). Immunoreactive proteins were detected using an enhanced chemiluminescence kit (PerkinElmer, Waltham, MA, USA), and bands quantified with the Quantity One software (version 4.0; Bio-Rad, Hercules, CA, USA) by densitometry, with $\beta$-actin used as a loading control. Normalized data are expressed as fold increases compared with SHAM or OVX control.

Statistical analysis. The analyses were conducted using SPSS (version 13.0; SPSS Inc., Chicago, IL, USA). The difference between the groups regarding the evaluated parameters was assessed by analysis of variance followed by the least significant difference test. The data of all groups passed the Kolmogorov-Smirnov test of normality. $\mathrm{P}<0.05$ was considered to indicate a statistically significant difference.

\section{Results}

Differentially expressed genes. In total, 380 differentially expressed genes ( $\geq 3$-fold) between the RDE and OVX groups were identified. Specifically, 205 genes were upregulated, and 175 downregulated (data not shown).

PPInetwork. Based on the differentially expressed genes (data not shown), a biological network showing protein-protein interactions was established. The PPI network was visualized using Cytoscape. In the PPI network, the nodes represent proteins, while the edges reflect the biological relationships between two given nodes. A total of 631 nodes and 1,273 edges were obtained in the PPI network (data not shown). 
Table I. Molecular clusters in the protein-protein interaction network.

\begin{tabular}{lll}
\hline Cluster & P-value & \multicolumn{1}{c}{ Members } \\
\hline Cluster A & 0.017641 & Cdk1 Hist1h1a Cdh1 Cdk2 Ccna1 Ccne1 \\
Cluster B & 0.025993 & Slc40a1 Kcnab2 Kcna2 Kcna1 Kcnab1 \\
Cluster C & 0.026880 & Dnmt1 Pcna Esr2 Nr0b2 \\
Cluster D & 0.049713 & Dnmt1 Pcna Esr2 Kctd10
\end{tabular}

A

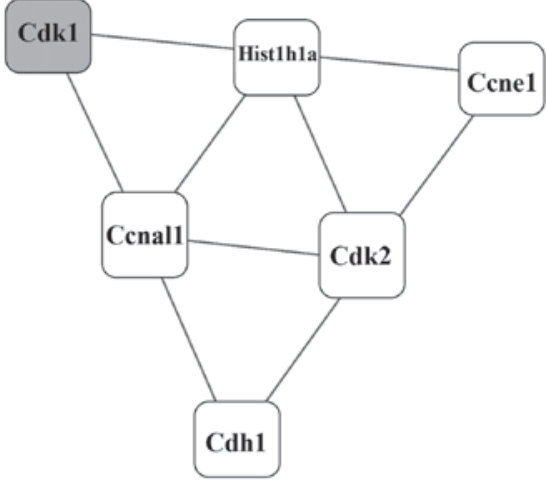

B

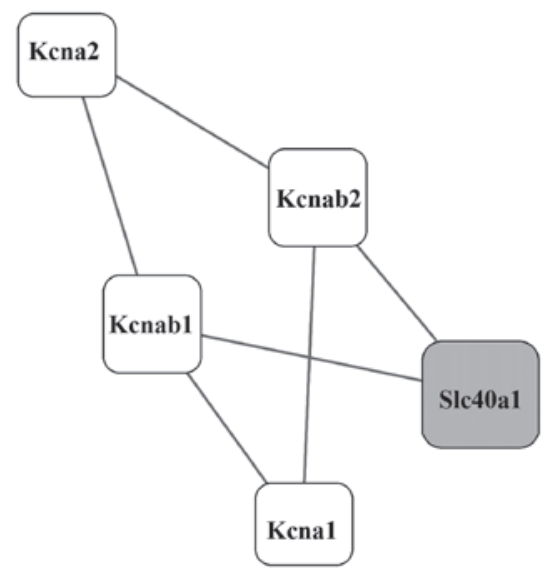

$\mathbf{C}$

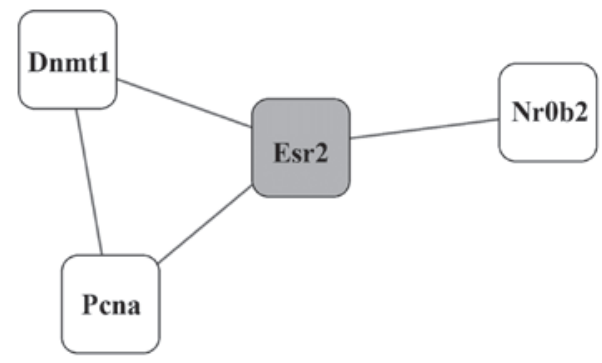

D

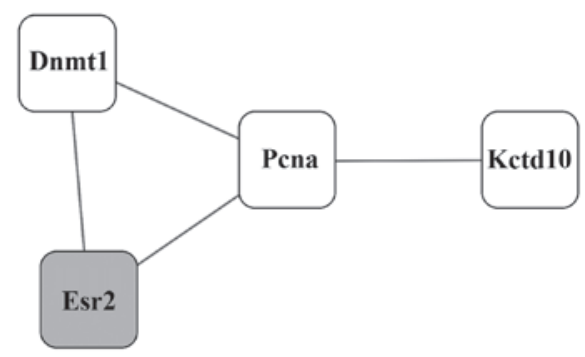

Figure 1. Four molecular clusters consisted of at least four nodes and one seed node in the protein-protein interaction network. Gray squares represent seed nodes, and white squares represent neighboring nodes. All edges represent interactions between the nodes. (A) Cluster A; (B) Cluster B; (C) Cluster C; (D) Cluster D.
Molecular clusters in the PPI network. Using the MCODE algorithm, four molecular clusters (A-D) in the PPI network were identified with smallest P-values, and were considered the significant molecular clusters (Table I and Fig. 1A-D).

Biological processes associated with molecular clusters in the PPI network. The DAVID tool was used to retrieve biological process information associated with the four molecular clusters. Of the four clusters, only two (A and B) were functionally annotated (Table II).

Biological pathways associated with molecular clusters in the PPI network. Table III summarizes the top IPA canonical pathways $(\mathrm{P}<0.001)$ which are associated with the molecular clusters in the PPI network and include the seed genes. Of the four clusters, only Cluster A was associated with biological pathways in the IPA pathway database. Table III shows that these pathways were predominantly associated with the cell cycle. Figs. 2-4 depict the three pathways involved in the cell cycle (displayed in Table III) with the seed genes are highlighted in gray.

Protein expression by western blotting. Protein expression of CDK1 was measured in alveolar bone of rats from the SHAM, OVX and RDE groups. Notably, changes in the level of CDK1 protein was in agreement with mRNA expression. Indeed, CDK1 was downregulated in RDE-treated rats compared with the OVX group (Fig. 5).

\section{Discussion}

In the present study, a huge PPI network was established based on the differentially expressed genes found in our previous study. To understand the complexity of this network, one of the most accepted methods was used, analyzing modules or molecular clusters. A molecular cluster is composed of a number of interrelated molecules, has a stable structure, and reflects a specific biological function. Table II shows the biological processes of two significant molecular clusters in the PPI network. Cluster A and B were shown to be associated with the cell cycle and ion transport, respectively.

In addition to biological function analysis, pathway prediction is another important method to extract potential signaling pathway information from molecular networks, and contributes to understanding the mechanisms of a given biological process (17). Table III presents the key canonical pathways of cluster A; the only significant molecular cluster in the generated PPI network. As shown in Table III, cluster A was associated with the cell cycle. As shown in Table III, clusters A 
Table II. Key biological processes associated with the clusters.

\begin{tabular}{|c|c|c|c|c|}
\hline Cluster & Biological process & Term & P-value & FDR \\
\hline \multirow[t]{3}{*}{ Cluster A } & GOTERM_BP_FAT & Cell division & $8.70 \times 10^{-4}$ & $1.0 \times 10^{-2}$ \\
\hline & GOTERM_BP_FAT & Response to drug & $4.20 \times 10^{-3}$ & $5.0 \times 10^{-2}$ \\
\hline & GOTERM_BP_FAT & Cell cycle & $8.20 \times 10^{-3}$ & $9.5 \times 10^{-2}$ \\
\hline \multirow[t]{5}{*}{ Cluster B } & GOTERM_BP_FAT & Metal ion transport & $1.20 \times 10^{-6}$ & $8.5 \times 10^{-4}$ \\
\hline & GOTERM_BP_FAT & Cation transport & $2.70 \times 10^{-6}$ & $1.9 \times 10^{-3}$ \\
\hline & GOTERM_BP_FAT & Potassium ion transport & $6.30 \times 10^{-6}$ & $4.4 \times 10^{-3}$ \\
\hline & GOTERM_BP_FAT & Ion transport & $9.20 \times 10^{-6}$ & $6.4 \times 10^{-3}$ \\
\hline & GOTERM_BP_FAT & Monovalent inorganic cation transport & $5.40 \times 10^{-5}$ & $3.7 \times 10^{-2}$ \\
\hline
\end{tabular}

FDR, false discovery rate.

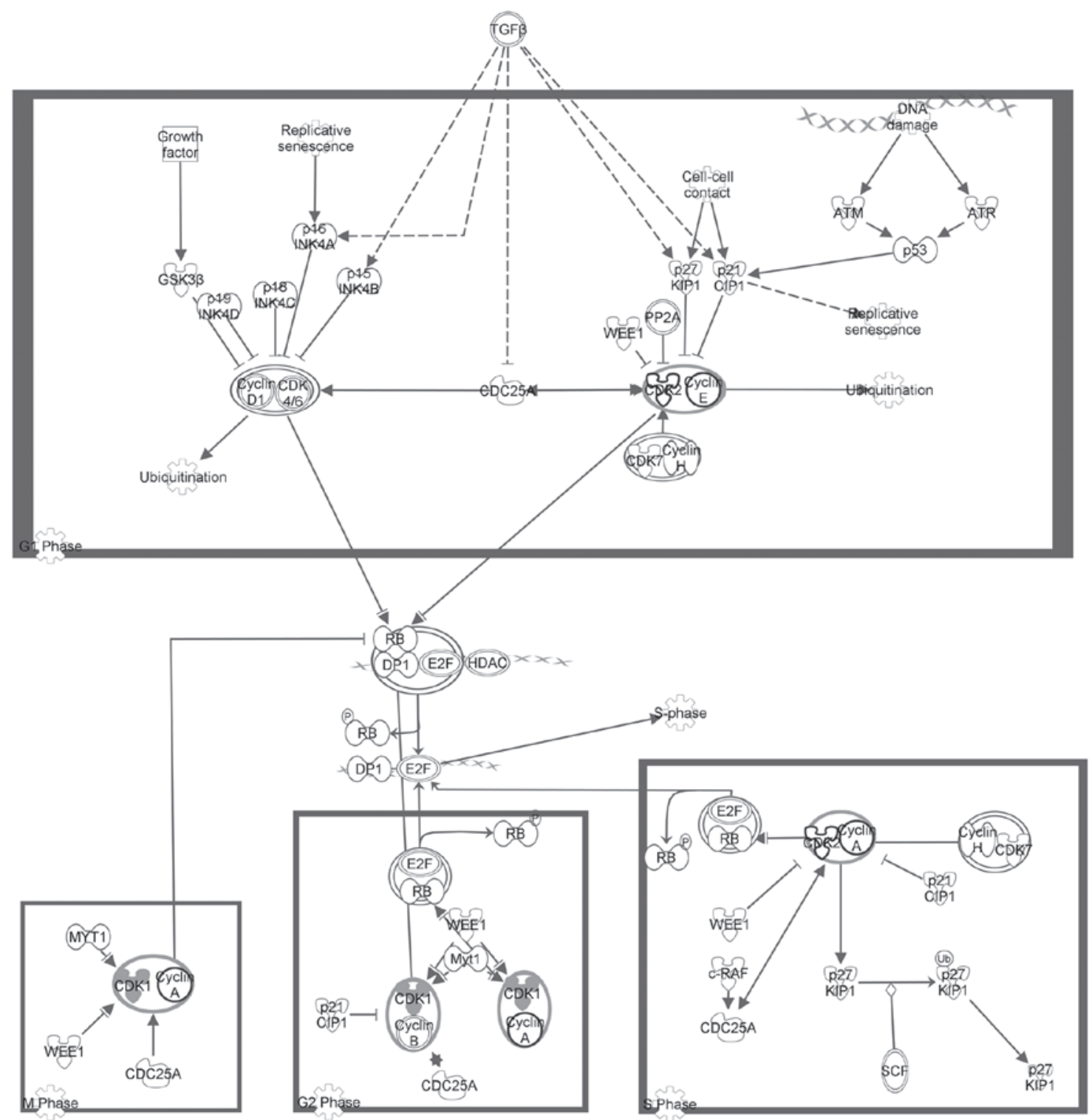

Figure 2. Cyclins and cell cycle regulation are associated with Cluster A. The downregulated seed gene in cluster appears is shown in gray, and the other genes in the cluster are shown by a bold line. The genes that are not specified and incorporated into the network through relationships are shown with a thin line.

and $\mathrm{C}$ were associated with the cell cycle and potassium ion channel, respectively.
Potassium channels are major intracellular signaling pathways at the bone cell surface, through which cell signals 
Table III. Key canonical pathways associated with the clusters.

\begin{tabular}{lll}
\hline Cluster & \multicolumn{1}{c}{ Pathways } & P-value \\
\hline Cluster A & Estrogen-mediated S-phase entry & $4.54 \times 10^{-5}$ \\
& $\begin{array}{l}\text { Cyclins and cell cycle regulation } \\
\text { Regulation of cellular mechanics }\end{array}$ & $1.73 \times 10^{-4}$ \\
& $\begin{array}{l}\text { by calpain protease } \\
\text { b.8. }\end{array}$ & \\
\hline
\end{tabular}

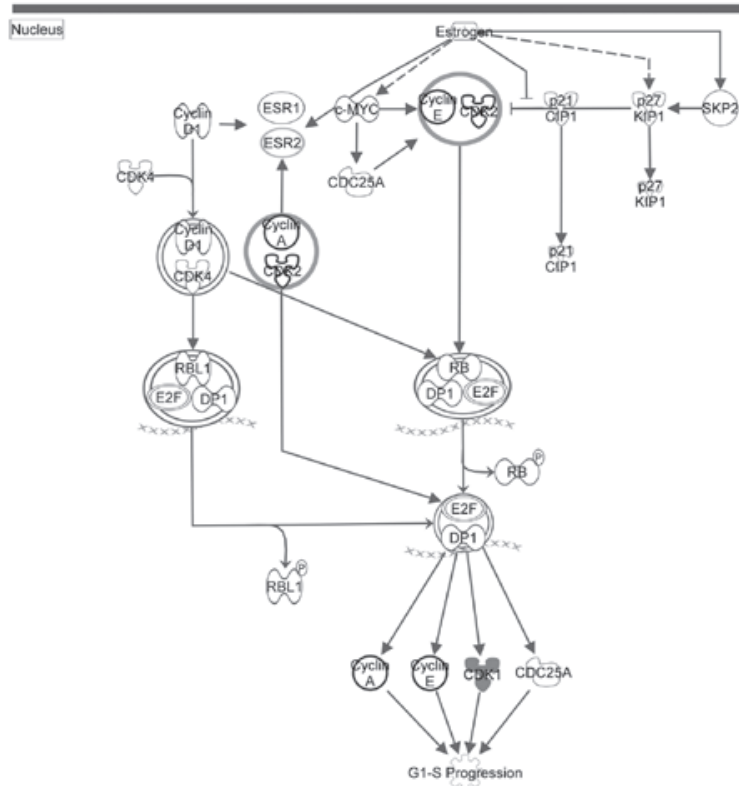

Figure 3. Estrogen-mediated S-phase entry associated with Cluster A. The downregulated seed gene in Cluster A is shown in gray, and the other genes in the cluster are shown with a bold line. The genes that are not specified and incorporated into the network through relationships are shown by a thin line.

are transferred across to the nuclear material for subsequent cellular activation (18). In osteoclasts, potassium ion channels include an inward rectifier channel regulated by $\mathrm{G}$ proteins, and a transient outward rectifier channel regulated by cell-matrix interactions and extracellular cations such as calcium and hydrogen (19). Solute carrier family 40 (SLC40A1) is a cell membrane protein involved in iron export from the cells to the blood (20). The relationship between SLC40A1 and potassium channels remains unknown, and it may be interesting to identify the role of SLC40A1 in regulating potassium channels.

As only cluster A was assigned both function and pathway annotation, it was further assessed. The cell cycle is modulated by multiple regulatory molecules, including cyclins and cyclin-dependent kinases (CDKs). CDKs are a family of protein kinases that are essential for cell cycle progression. They bind to specific cyclins and are further activated (21). CDK1 is known to be a highly conserved protein, and functions as a serine/threonine kinase. CDK1 binds to cyclins A and B, and is involved in modulating the S, G2 and M phases of the cell cycle (22).

The rat OVX model is the most commonly used and extensively studied animal model of early postmenopausal osteoporosis. Unlike late postmenopausal osteoporosis (23), early postmenopausal osteopenia, including alveolar bone loss,

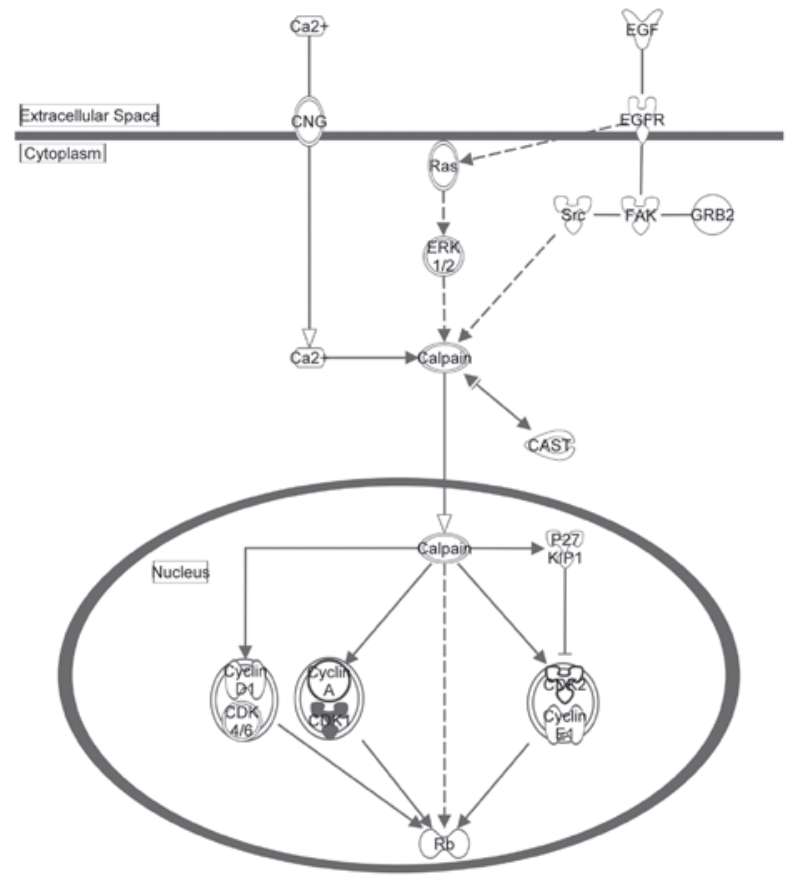

Figure 4. Regulation of cellular mechanics by calpain protease associated with Cluster A. The downregulated seed gene in Cluster A appears in gray, and the other genes in the cluster are shown with a bold line. The genes that are not specified and incorporated into the network through relationships are shown with a thin line.

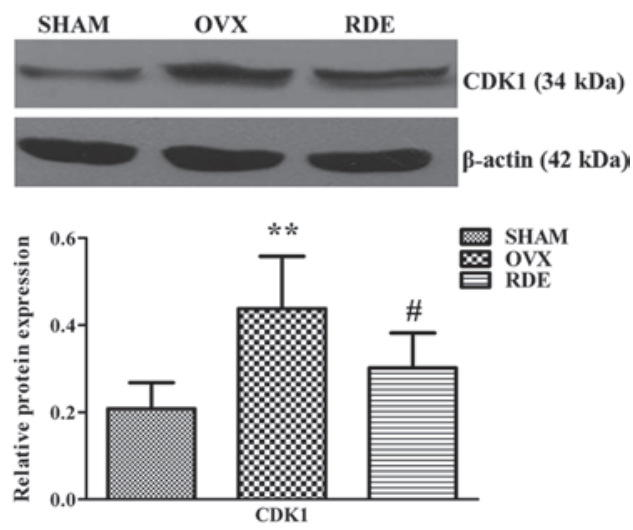

Figure 5. Validation of the effect of RDE on the expression of CDK1 by western blotting. $\beta$-actin was employed as the housekeeping gene. Representative blots are shown (left). Band intensities were quantified from six independent samples using densitometry (right). ${ }^{* *} \mathrm{P}<0.01$ vs. the SHAM group; ${ }^{\text {P }}<0.01$ vs. the OVX group. RDE, Rhizoma Dioscoreae; CDK1, cyclin-dependent kinase 1; OVX, ovariectomized.

is characterized by high bone turnover that can reproducibly be induced in the OVX animal model (24). In high-turnover bone loss, the marked increase in the bone turnover rate is triggered by an imbalance between bone resorption and formation, with resorption exceeding formation (25). OVX may raise the number of pre-osteoblasts in the $S$ phase due in part to the inhibition of osteoprogenitor cell proliferation in rodent models $(26,27)$. With regard to osteoclasts, studies have indicated that RANKL induces RAW264.7 cell proliferation and promotes cell cycle transition (28), whereas Herba Epimedii flavonoids may suppress this process (29). Thus, excessive bone formation and resorption in high-turnover 
bone remodeling are associated with promoting cell cycle progression.

The $\mathrm{S}$ phase is an important process common to all cell types during which chromosomes are replicated; loss of normal replication control is a hallmark of cancer (30). In breast cancer cells, estrogen and its receptors can stimulate G1-S phase transition via cyclins or CDKs $(31,32)$; specifically, CDK2 and CDK1 are essential in S phase control (33). In the present study, it was demonstrated that RDE was able to inhibit the expression of CDK1 in the alveolar bone of OVX rats. The retarding cell cycle progression in $\mathrm{S}$ phase may be one of mechanisms by which RDE protects against alveolar bone loss.

Calpains are non-lysosomal, calcium-dependent cysteine proteases, which are highly conserved and found in cells of organisms, ranging from mammals to Drosophila. They are pivotal proteases in limited proteolysis of various structural proteins, regulatory proteins and the tumor-suppressor protein retinoblastoma $(\mathrm{Rb})$. Enhanced calpain activity is also associated with cell cycle progression. Calpain promotes transformed cells to go through G1, enhances hyper-phosphorylation of the $\mathrm{Rb}$ protein and increases protein expression levels of cyclin D, cyclin A, CDK2 and CDK1 (34). Calpains are involved in bone remodeling, namely cytoskeletal remodeling, integrin-mediated cell migration, cell differentiation and apoptosis (35-38). We speculated that the retarding cell cycle progression in G1 phase may be another mechanism by which RDE protects against alveolar bone loss.

GO and pathway analysis data showed that the cluster A was associated with cell cycle regulation and was the most important in the PPI network. CDK1 in this cluster is a key positive cell cycle regulator; notably, CDK1 was shown to be downregulated at the gene level following RDE treatment in our previous study (10). Here, the change in CDK1 protein levels corroborated gene expression data (Fig. 5). Based on the results described above, it was inferred that RDE may prevent alveolar bone loss by downregulating CDK 1 and further inhibiting cell cycle progression in alveolar bone tissues.

A few limitations of this study should be mentioned. MCODE used in the present study is only one of the multiple algorithms that can be used to determine molecular clusters in PPI networks, the other molecular clusters or functional modules with important biological significance may be identified with the use of the other algorithms.

Using PPI network analysis, this study predicted that delayed cell cycle progression in bone remodeling via CDK1 downregulation may be a mechanism behind the anti-osteopenic effect of RDE on alveolar bone. Studies are warranted to further investigate the mechanisms underlying the anti-osteopenic effect of RDE.

\section{Acknowledgements}

This study was supported by the National Natural Science Foundation of China (grant nos. 81102680 and 81473450), the Fundamental Research Funds for the Central Public Welfare Research Institutes (grant no. YZ-1409) and the Beijing Foundation for Science and Technology Development of Traditional Chinese Medicine (grant no. JJ2015-54).

\section{References}

1. Sultan N and Rao J: Association between periodontal disease and bone mineral density in postmenopausal women: A cross sectional study. Med Oral Patol Oral Cir Bucal 16: e440-e447, 2011.

2. Streckfus CF, Johnson RB, Nick T, Tsao A and Tucci M: Comparison of alveolar bone loss, alveolar bone density and second metacarpal bone density, salivary and gingival crevicular fluid interleukin- 6 concentrations in healthy premenopausal and postmenopausal women on estrogen therapy. J Gerontol A Biol Sci Med Sci 52: M343-M351, 1997.

3. CivitelliR,PilgramTK,DotsonM,MuckermanJ,LewandowskiN, Armamento-Villareal R, Yokoyama-Crothers N, Kardaris EE, Hauser J, Cohen S and Hildebolt CF: Alveolar and postcranial bone density in postmenopausal women receiving hormone/estrogen replacement therapy: A randomized, double-blind, placebo-controlled trial. Arch Intern Med 162: 1409-1415, 2002.

4. Palomo L, Bissada NF and Liu J: Periodontal assessment of postmenopausal women receiving risedronate. Menopause 12: 685-690, 2005.

5. Liu J, Cao Z and Li C: Intermittent PTH administration: A novel therapy method for periodontitis-associated alveolar bone loss. Med Hypotheses 72: 294-296, 2009.

6. Strom BL, Schinnar R, Weber AL, Bunin G, Berlin JA, Baumgarten M, DeMichele A, Rubin SC, Berlin M, Troxel AB and Rebbeck TR: Case-control study of postmenopausal hormone replacement therapy and endometrial cancer. Am J Epidemiol 164: 775-786, 2006.

7. Woo SB, Hellstein JW and Kalmar JR: Narrative [corrected] review: Bisphosphonates and osteonecrosis of the jaws. Ann Intern Med 144: 753-761, 2006.

8. Rizzoli R, Reginster JY, Boonen S, Bréart G, Diez-Perez A, Felsenberg D, Kaufman JM, Kanis JA and Cooper C: Adverse reactions and drug-drug interactions in the management of women with postmenopausal osteoporosis. Calcif Tissue Int 89: 91-104, 2011.

9. Clemett D and Spencer CM: Raloxifene: A review of its use in postmenopausal osteoporosis. Drugs 60: 379-411, 2000.

10. Zhang Z, Xiang L, Bai D, Wang W, Li Y, Pan J, Liu H, Wang S, Xiao GG and Ju D: The protective effect of Rhizoma Dioscoreae extract against alveolar bone loss in ovariectomized rats via regulating Wnt and p38 MAPK signaling. Nutrients 6: 5853-5870, 2014

11. Wu Z, Zhao X and Chen L: Identifying responsive functional modules from protein-protein interaction network. Mol Cells 27: 271-277, 2009.

12. Real-Chicharro A, Ruiz-Mostazo I, Navas-Delgado I, Kerzazi A, Chniber O, Sánchez-Jiménez F, Medina MA and Aldana-Montes JF: Protopia: A protein-protein interaction tool. BMC Bioinformatics 10 (Suppl 12): S17, 2009.

13. Martin A, Ochagavia ME, Rabasa LC, Miranda J, Fernandez-de-Cossio J and Bringas R: BisoGenet: A new tool for gene network building, visualization and analysis. BMC Bioinformatics 11: 91, 2010.

14. Shannon P, Markiel A, Ozier O, Baliga NS, Wang JT, Ramage D, Amin N, Schwikowski B and Ideker T: Cytoscape: A software environment for integrated models of biomolecular interaction networks. Genome Res 13: 2498-2504, 2003.

15. Bader GD and Hogue CW: An automated method for finding molecular complexes in large protein interaction networks. BMC Bioinformatics 4: 2, 2003.

16. Huang da W, Sherman BT and Lempicki RA: Systematic and integrative analysis of large gene lists using DAVID bioinformatics resources. Nat Protoc 4: 44-57, 2009.

17. Gomez SM: Prediction of protein-protein interaction networks. Curr Protoc Bioinformatics Chapter 8: Unit 8.2, 2003.

18. McDonald F: Ion channels in osteoblasts: A story of two intracellular organelles. Surgeon 2: 63-69, 2004.

19. Supanchart $C$ and Kornak U: Ion channels and transporters in osteoclasts. Arch Biochem Biophysics 473: 161-165, 2008.

20. Ward DM and Kaplan J: Ferroportin-mediated iron transport: Expression and regulation. Biochim Biophys Acta 1823: 1426-1433, 2012.

21. Li JM and Brooks G: Cell cycle regulatory molecules (cyclins, cyclin-dependent kinases and cyclin-dependent kinase inhibitors) and the cardiovascular system; potential targets for therapy? Eur Heart J 20: 406-420, 1999. 
22. Dorée M and Hunt T: From Cdc2 to Cdk1: When did the cell cycle kinase join its cyclin partner? J Cell Sci 115: 2461-2464, 2002.

23. Tchetina EV, Maslova KA, Krylov MY and Myakotkin VA Association of bone loss with the upregulation of survival-related genes and concomitant downregulation of Mammalian target of rapamycin and osteoblast differentiation-related genes in the peripheral blood of late postmenopausal osteoporotic women. J Osteoporos 2015: 802694, 2015.

24. Wronski TJ, Lowry PL, Walsh CC and Ignaszewski LA: Skeletal alterations in ovariectomized rats. Calcif Tissue Int 37: 324-328, 1985.

25. Garnero P, Sornay-Rendu E, Chapuy MC and Delmas PD: Increased bone turnover in late postmenopausal women is a major determinant of osteoporosis. J Bone Miner Res 11: 337-349, 1996.

26. Turner RT, Backup P, Sherman PJ, Hill E, Evans GL and Spelsberg TC: Mechanism of action of estrogen on intramembranous bone formation: Regulation of osteoblast differentiation and activity. Endocrinology 131: 883-889, 1992.

27. Orlić I, Borovecki F, Simić P and Vukicević S: Gene expression profiling in bone tissue of osteoporotic mice. Arh Hig Rada Toksikol 58: 3-11, 2007.

28. Li CH, Zhao JX, Sun L, Yao ZQ, Deng XL, Liu R and Liu XY: AG490 inhibits NFATc1 expression and STAT3 activation during RANKL induced osteoclastogenesis. Biochem Biophys Res Commun 435: 533-539, 2013.

29. Zhang D, Zhang J, Fong C, Yao X and Yang M: Herba epimedii flavonoids suppress osteoclastic differentiation and bone resorption by inducing $\mathrm{G} 2 / \mathrm{M}$ arrest and apoptosis. Biochimie 94 2514-2522, 2012.

30. Wuarin J and Nurse P: Regulating S phase: CDKs, licensing and proteolysis. Cell 85: 785-787, 1996.
31. Prall OW, Sarcevic B, Musgrove EA, Watts CK and Sutherland RL: Estrogen-induced activation of Cdk4 and Cdk2 during G1-S phase progression is accompanied by increased cyclin D1 expression and decreased cyclin-dependent kinase inhibitor association with cyclin E-Cdk2. J Biol Chem 272: 10882-10894, 1997.

32. Vondracek J, Kozubik A and Machala M: Modulation of estrogen receptor-dependent reporter construct activation and G0/G1-S-phase transition by polycyclic aromatic hydrocarbons in human breast carcinoma MCF-7 cells. Toxicol Sci 70: 193-201, 2002.

33. Hayashi S and Yamaguchi M: Kinase-independent activity of $\mathrm{Cdc} 2 /$ cyclin A prevents the $\mathrm{S}$ phase in the Drosophila cell cycle. Genes Cells 4: 111-122, 1999.

34. Suzuki K, Hata S, Kawabata Y and Sorimachi H: Structure, activation, and biology of calpain. Diabetes 53 (Suppl 1): S12-S18, 2004.

35. Hayashi M, Koshihara Y, Ishibashi H, Yamamoto S, Tsubuki S, Saido TC, Kawashima S and Inomata M: Involvement of calpain in osteoclastic bone resorption. J Biochem 137: 331-338, 2005.

36. Murray EJ, Tram KK, Spencer MJ, Tidball JG, Murray SS and Lee DB: PTH-mediated osteoblast retraction: Possible participation of the calpain pathway. Miner Electrolyte Metab 21: 184-188, 1995.

37. Shimada M, Greer PA, McMahon AP, Bouxsein ML and Schipani E: In vivo targeted deletion of calpain small subunit, Capn 4, in cells of the osteoblast lineage impairs cell proliferation, differentiation and bone formation. J Biol Chem 283: 21002-21010, 2008.

38. Murray SS, Grisanti MS, Bentley GV, Kahn AJ, Urist MR and Murray EJ: The calpain-calpastatin system and cellular proliferation and differentiation in rodent osteoblastic cells. Exp Cell Res 233: 297-309, 1997. 